\title{
Fitness ecológico
}

\author{
Ecological fitness
}

\author{
SANTIAGO GINNOBILI \\ Universidad de Buenos Aires - Universidad Nacional de Quilmes - CONICET \\ (Argentina)
}

Recibido: 9-1-2013

Aprobado definitivamente: 25-2-2013

\section{RESUMEN}

Existe un acuerdo relativo en la necesidad de distinguir dos usos del término «fitness»: el ecológico y el de la genética de poblaciones. Algunos consideran que el segundo ha venido a reemplazar al primero. Otros que el fitness ecológico tiene cierta capacidad explicativa de la que el segundo carece. Estos últimos autores han intentado dar respuesta a cómo es que el fitness ecológico se relaciona con las propiedades particulares de los organismos, siendo estas tan heterogéneas. En este trabajo intentaré dar una respuesta más adecuada a esta cuestión, utilizando el marco conceptual de la metateoría estructuralista.

\section{PALABRAS CLAVE}

FITNESS, SUPERVENIENCIA, SELECCIÓN NATURAL, ESTRUCTURALISMO METATEÓRICO, INTERPRETACIÓN PROPENSIONISTA DEL FITNESS

\section{ABSTRACT}

There is relative agreement on the need to distinguish two different uses of the term «fitness»: the ecological fitness and the population genetics fitness. Many consider that the latter has come to replace the former. Others think that the ecological fitness has certain explanatory power that the population genetics fitness lacks of. Among the last ones, many have tried to give response to how ecological fitness relates to organism's properties, especially because of their being so heterogeneous. In this paper I will try to give a better answer to this matter using the conceptual framework of metatheoretical structuralism.

\section{KEYWORDS}

FITNESS, SUPERVENIENCE, NATURAL SELECTION, METATHEORETICAL STRUCTURALISM, PROPENSITY INTERPRETATION OF FITNESS

(C) Contrastes. Revista Internacional de Filosofía: Suplemento 18 (2013), pp. 83-97. ISSN: 1136-9922

Departamento de Filosofía, Universidad de Málaga, Facultad de Filosofía y Letras Campus de Teatinos, E-29071 Málaga (España) 
PoCo HA SIDO TAN DISCUTIDO EN EL MARCO DE LA FILOSOFÍA DE LA BIOLOGíA como el concepto de fitness. Existe, sin embargo, un acuerdo generalizado en que es necesario desambiguar al menos dos sentidos con los que el término «fitness» es utilizado: el fitness ecológico y el fitness de la genética de poblaciones. Presentaré esta distinción en el apartado I. Algunos autores consideran que el fitness de la genética de poblaciones viene a reemplazar al ecológico, propuesto en el marco de la teoría de la selección natural (en adelante TSN). En este mismo sentido, se considera que la TSN cualitiativa propuesta por Darwin ha sido reemplazada por una TSN cuantitativa en el marco de la genética de poblaciones (p.e. Ariew \& Lewontin 2004). En otras oportunidades he intentado defender en qué sentido esta idea es incorrecta (Ginnobili 2010b) en consonancia con otros autores que consideran que TSN es una teoría independiente de la genética de poblaciones, e intentan reconstruirla brindando de este modo una elucidación del fitness ecológico (p.e. Brandon 1978; Endler 1986; Williams 1970). Tales autores se enfrentan explícitamente a un problema e intentan elaborar soluciones: cómo dar cuenta del modo en que el concepto de fitness se relaciona con el conjunto extremadamente heterogéneo de capacidades de físicas de los organismos que afectan el éxito reproductivo. Trataré esta cuestión en II. Rosenberg, por ejemplo, apela al concepto de superveniencia (Rosenberg 1978), mientras que Brandon, a la interpretación propensionista de la probabilidad (Brandon 1980, 1990). Considero que las soluciones ofrecidas no son satisfactorias y que el problema puede ser tratado más adecuadamente ofreciendo reconstrucciones más adecuadas de TSN y apelando al aparato conceptual del estructuralismo metateórico (Balzer \& Moulines 1996; Balzer, Moulines \& Sneed 1987; Diez \& Lorenzano 2002; Moulines 1982; Moulines 1991). En III presentaré dos sentidos en que las recontrucciones de TSN deben completarse, utilizando como ejemplo la reconstrucción de TSN brindada por Brandon. En IV introduciré elementos básicos del estructuralismo metateórico que permitirán dar una solución más satisfactoria al problema que nos ocupa. Esta discusión permitirá comprender mejor el estatus del fitness ecológico y el modo en que se aplica, mostrando además, que TSN no es diferente de otras teorías de la misma y otras disciplinas, y en consecuencia, que no es necesario apelar a ninguna solución específica peculiar (como la superveniencia) para salvaguardar su capacidad explicativa.

\section{Dos FITNESS}

Varios autores han señalado la existencia de dos tipos de fitness. Sober distingue dos formas de concebir la selección natural, desde la genética de poblaciones y a través de las condiciones ecológicas que la producen (esto se corresponde con dos formas de calcular el fitness, a través de sus consecuencias o a través de las propiedades físicas que lo causan) (Sober 1993, pp. 13-59). 
Matthen \& Ariew distinguen entre el fitness vernáculo y el predictivo (Matthen \& Ariew 2002). El primero se basaría en las habilidades de los organismos para sobrevivir y reproducirse, y el segundo sería la medida estadística del cambio evolutivo, o la propensión a ser representado en futuras generaciones. Ariew \& Lewontin distinguen, por su parte, entre el fitness darwiniano, al que caracterizan como una metáfora inexacta de cómo el organismo calza en su ambiente, y lo contraponen al fitness reproductivo, calculado matemáticamente en la genética de poblaciones (Ariew \& Lewontin 2004). Pigliucci \& Kaplan, por su parte, distinguen ente un fitness informal y uno formal, y consecuentemente, entre una selección natural informal y una formal (Pigliucci \& Kaplan 2006, pp. 17-31). La idea es análoga. El fitness informal es acerca de la relación entre un rasgo y su relación con un ambiente y cómo afecta eso al éxito reproductivo del organismo que lo posee. El fitness formal es una propiedad estadística de las poblaciones que se define a partir de las tasas medias de subpoblaciones distinguidas por la posesión de una variación de un rasgo. Finalmente, en la entrada correspondiente a fitness en The Stanford Encyclopedia of Philosophy, Rosenberg y Bouchard distinguen entre el fitness ecológico del fitness de la genética de poblaciones. El primero tendría que ver con la capacidad del organismo de resolver problemas de diseño propuestos por el ambiente, mientras que el segundo con las tasas de reproducción genéticas (Rosenberg \& Bouchard 2009).

Una vez hecha esta distinción, existe la tendencia en algunos casos de considerar que el fitness de la genética de poblaciones provee una versión más exacta y cuantitativa del fitness ecológico. Lo que se presupone por detrás de esta idea es que la selección natural se define únicamente a partir de diferencias en el éxito de distintos organismos en la reproducción diferencial. Si esto fuese así, la genética de poblaciones, por permitir determinar con bastante precisión bajo ciertos supuestos que la frecuencia génica en una población no es la esperada, permitiría cuantificar la acción de la selección natural.

Así, por ejemplo, en su conocido libro de texto sobre evolución, Futuyma afirma: «Si los alelos difieren en sus tasas de reemplazo, sus frecuencias pueden cambiar. Este fenómeno es llamado 'selección'» (Futuyma 1986, pp. 86-87). Por otro lado, y en una disciplina diferente, el reconocido historiador de la ciencia Jean Gayon dice: «[Con el descubrimiento de Hardy y Weimberg] la selección natural no es más un principio fundamental sino un parámetro que mide una de las tantas fuerzas de cambio» (Gayon 1998, p. 321). Esto parece presupuesto también por ciertos autores que otorgan un lugar central o fundamental a la genética de poblaciones (Ruse 1973, cap. 4) y parece estar presupuesto, también, en autores que pretenden haber reconstruido la teoría evolutiva reconstruyendo sólo la genética de poblaciones o alguna parte de ella (p.e. Barbadilla 1990; Lloyd 1994; Thompson 1989). También es un presupuesto de los que pretenden 
elucidar el concepto de fitness a partir de la reconstrucción de la teoría de la evolución como una teoría de fuerzas (Sober 1993, pp. 13-59).

Algunos autores se oponen a que el concepto de fitness de la selección natural sea elucidado por su lugar en la genética de poblaciones (Glymour 2006, p. 388; Rosenberg 1994, pp. 110-111). Ha sido señalado, en consonancia con estos puntos de vista, el carácter restrictivo del concepto de fitness de la genética de poblaciones, defendiendo al concepto de fitness ecológico, como más explicativo y abarcativo (Bouchard 2011; Peacock 2011). El desafío frente a las posiciones críticas del fitness ecológico, que lo consideran informal, presistemático o vago, consiste en mostrar su inserción en una teoría bien reconstruida mostrando el modo en que se aplica a los casos pretendidos.

\section{FITNESS ECOLÓGICO Y LAS PROPIEDADES FÍSICAS DE LOS ORGANISMOS}

Existe un problema que enfrentan las reconstrucciones de TSN que la presentan como una teoría independiente de la genética de poblaciones y que permitirían por tanto elucidar el concepto de fitness ecológico, que consiste en dar cuenta de cómo se relacionan las propiedades específicas de los organismos con el fitness (en adelante con «fitness», me referiré al fitness ecológico salvo que lo aclare) y con el éxito reproductivo. Suele considerarse: (i) que existe una relación entre el fitness y el éxito reproductivo diferencial; (ii) que el fitness no puede reducirse al concepto de éxito reproductivo diferencial (evadiendo el problema de la tautologicidad); y (iii) que el fitness tiene algún tipo de relación con capacidades físicas particulares de los organismos. Todos los que defienden el concepto de fitness ecológico hacen malabares para lograr satisfacer esas condiciones de manera explícita en sus elucidaciones. Un ejemplo representativo de tratamiento de esta problemática es la propuesta de Rosenberg, de tratar la relación entre fitness y las capacidades físicas de los organismos a partir del concepto de superveniencia (Rosenberg 1978, 1985,pp. 164-169). Efectivamente, si el concepto de fitness es explicativo de las diferencias en el éxito reproductivo, entonces tiene que implicar algo más que las meras diferencias en el éxito reproductivo. Rosenberg se pregunta cómo podría el fitness relacionarse con las capacidades físicas de los organismos (1978, p. 371), si es posible que dos organismos distintos tengan el mismo fitness, uno porque evade predadores por camuflaje, otro por el vuelo. El fitness de los organismos se encuentra interconectado con un número vasto de capacidades físicas y condiciones ambientales diferentes, por lo que se volvería imposible, según Rosenberg, establecer incluso una pequeña proporción de las conexiones nomológicas entre un fitness dado y todas las diferentes propiedades y relaciones de los organismos que lo pueden afectar (Sober coincide con Rosenberg en este punto, Sober 1993, p. 48). Esto implica, según él, que el fitness sólo pueda ser medido a través de las tasas de 
reproducción. La forma en que Rosenberg salvaguarda la capacidad explicativa de TSN es apelando al concepto de superveniencia (Davidson 1970):

El concepto de fitness superviene sobre las propiedades manifiestas de los organismos, sus propiedades anatómicas, fisiológicas, comportamentales y relativas al ambiente. Y este hecho solo explica el poder explicativo y la recalcitrancia empírica simultáneas del concepto de fitness (Rosenberg 1978, p. 372).

El concepto de superveniencia permite sostener que las propiedades supervenientes no son reducibles a las fisicalistas, pero son dependientes en el sentido de que no puede haber dos eventos semejantes en todos sus aspectos físicos pero diferentes al respecto de la característica superveniente. ${ }^{1}$

El problema, como decíamos, consiste en relacionar al concepto de fitness con el éxito reproductivo, por un lado, y con las capacidades físicas de los organismos, por el otro, conservando la fuerza explicativa de TSN (Rosenberg 1983460-461). Este problema también es tratado por Brandon (1990, pp. 1213). Su solución, siguiendo a la de Mills \& Beatty (1979), consiste en apelar a la interpretación del fitness como propensión. Esto evita que el enunciado «los más aptos dejan más descendencia» sea tautológico, porque una cosa es la descendencia que de hecho se deja y otra la propensión o disposición a dejar cierta cantidad de descendencia. Se explica el éxito en la reproducción diferencial apelando a la propensión de dejar cierta cantidad de descendencia de los organismos en cuestión, como se explica que cierta porción de sal se disuelve por ser soluble (Mills \& Beatty 1979, p. 270). Rosenberg tiene razón al dudar de la fuerza explicativa de ese enunciado (que compara con la explicación de que el opio causa sueño a causa de su virtud dormitiva) (Rosenberg 1985, p. 160), en tanto no se señale el mecanismo sobre el cuál la disposición superviene. Es claro sin embargo, que esto mismo es tenido en cuenta por Brandon, puesto que la idea de la interpretación propensionista del fitness es que la propensión se sustenta sobre ciertas propiedades físicas. Tanto Brandon como Rosenberg consideran que la apelación a tales propiedades físicas de los organismos es la clave para salvaguardar la explicatividad de TSN.

No niego que la noción de superveniencia pueda ser útil para discutir ciertas cuestiones metafísicas acerca de la reducción en biología, discusiones que interesan particularmente a Rosenberg (2008,pp. 96-126). Esta noción, justamente, permite sostener una posición fisicalista, sin por ello comprometerse con un punto de vista reduccionista respecto de las propiedades supervenientes (Perez 1996). El problema que estamos enfrentando, sin embargo, no es metafísico,

1 Sober también da un tratamiento de esta cuestión apelando a la superveniencia (Sober 1993, pp. 47-59). 
y podría plantearse de este modo: si el fitness sólo se calcula a través del éxito reproductivo real de los diferentes organismos, qué papel juegan en la explicación las propiedades físicas de los organismos y cómo se relacionan con el fitness. La solución a la relación entre las propiedades de los organismos y el éxito en la reproducción diferencial en las aplicaciones que hacen los biólogos de TSN no es un problema metafísico. La cuestión en juego es cómo relacionan estos diferentes conceptos en las explicaciones dadas por científicos en su práctica habitual. La respuesta metafísica no puede, en este sentido, ser ni pertinente ni, en consecuencia, satisfactoria. Si las propiedades físicas de los organismos tienen un rol explicativo en las aplicaciones de TSN, entonces, tienen que aparecer de algún modo en una reconstrucción adecuada de TSN.

\section{INADECUACIÓN DE LAS RECONSTRUCCIONES DISPONIBLES DE LA TEORÍA DE LA SELECCIÓN NATURAL}

Considero que la problemática señalada en el apartado anterior puede resolverse apelando a una reconstrucción más completa de TSN. Utilizaré la propuesta de Brandon para ejemplificar este punto.

Brandon (1990) presenta TSN a partir de su ley fundamental:

Si $a$ está mejor adaptado que $b$ a un ambiente $E$, entonces (probablemente) $a$ tendrá un éxito reproductivo mayor que $b$ en $E .^{2}$

Esta ley permitiría por medio de las especificaciones correctas llegar a las propiedades específicas. El ejemplo brindado por Brandon es el de la famosa Biston betularia. Por instanciación de la ley fundamental es possible obtener: «Si $a$ tiene las alas más oscuras que $b$ (en $E$ ) entonces (probablemente) $a$ dejará más descendencia que $b$ (en $E$ )» (Brandon 1980, 1990, p. 23). La forma en que se obtiene el enunciado es reemplazando la propensión a dejar más descendencia, por una de sus realizaciones físicas, en este caso, el color de las alas.

Es posible realizar dos comentarios relevantes al tema que estamos tratando, respecto de esta forma de pensar TSN propuesta por Brandon.

Por una parte, la instanciación de los diferentes componentes de esta ley fundamental no brinda la explicación darwiniana completa. En ésta se explicita el modo en el cuál la coloración de las alas es relevante para la supervivencia. La explicación completa tendría más componentes:

2 Las diferencias terminológicas y la ambigüedad de los términos de la biología evolutiva traen más de un dolor de cabeza al filósofo de la biología en su iniciación. Brandon, no utiliza el término «fitness» para referirse al fitness ecológico. Pero lo que en esta ley aparece como «más adaptado» puede leerse como «con un mayor fitness ecológico» sin problemas. 
Las polillas de alas de color más oscuro se mimetizan mejor con su ambiente a los ojos de sus predadores, mejorando su supervivencia, mejorando en consecuencia su éxito en la reproducción diferencial.

En los trabajos de Kettlewell $(1955,1956)$ era esencial no únicamente encontrar la correlación entre éxito reproductivo diferencial y el color de las alas, sino, y a eso se dedicaron muchos de los experimentos, determinar que esa correlación se debía al mimetismo frente a sus predadores naturales y a la mejora en la supervivencia que este producía.

En otras oportunidades intenté mostrar en qué sentido las reconstrucciones disponibles no explicitaban todos los conceptos involucrados en una explicación seleccionista darwiniana, y brindé una reconstrucción que considero más adecuada de TSN (Ginnobili 2010a, 2011b, 2012). La ley fundamental tendría más componentes habitualmente dejados de lado. Un esbozo informal de ella sería el siguiente:

Los organismos que portan un rasgo que cumple más efectivamente una función mejoran su fitness, tendiendo a mejorar, en consecuencia, si el rasgo es heredable, su éxito reproductivo diferencial.

Al especificar cada uno de los componentes de esta ley fundamental, sí se obtiene la explicación completa (o al menos más completa que la esbozada por Brandon) del mayor éxito reproductivo de las polillas de alas más oscuras. ${ }^{3}$ Más adelante señalaré en qué sentido tomar en consideración esta cuestión es importante para una mejor presentación de la forma en que el fitness ecológico se relaciona con las propiedades físicas de los organismos.

El segundo comentario relevante que se puede realizar acerca de la reconstrucción de Brandon tiene que ver con la forma en que se obtienen las aplicaciones a partir de la ley fundamental, por una instanciación directa. Generalmente, las leyes fundamentales, al menos, las de las teorías más unificadoras, no se aplican de manera directa a la realidad, sino a partir de leyes especiales en las que se especifican lo que en ellas se encuentra inespecificado. Este hecho fue señalado con claridad por Kuhn (1970, pp. 182-189) y es retomado en el marco del estructuralismo metateórico (Balzer, Moulines \& Sneed 1987, pp. 167-203). Así, la ley fundamental de la mecánica clásica no se aplica sino a través de leyes especiales en las que se establecen los tipos de fuerzas en juego y la estructura matemática específica de dichas fuerzas. En la reconstrucción de Brandon, la

3 Casanueva, por su parte, ha ofrecido una reconstrucción algo diferente de la propuesta por mí, pero que en lo fundamental coincide (Casanueva 2011). La ley fundamental de TSN tendría al menos los tres componentes señalados. 
ley fundamental de TSN parece una ley estadística aislada, pero no lo es. Endler nos da una pista de en qué podrían consistir las leyes especiales de TSN. Para Endler el fitness no es únicamente una propensión a dejar más descendencia, sino una relación entre un rasgo y la habilidad para encontrar pareja, la fertilidad, la fecundidad y/o la supervivencia, que afecta a la reproducción diferencial (Endler 1986, pp. 3-15, 1992). Es decir, el fitness en sus diferentes aplicaciones varía entre una de las habilidades señaladas. El gráfico con el que Endler ilustra las diferentes subdivisiones de TSN puede ser interpretado como las diferentes leyes especiales en las que la ley fundamental presentada puede especializarse (ver fig. 1).

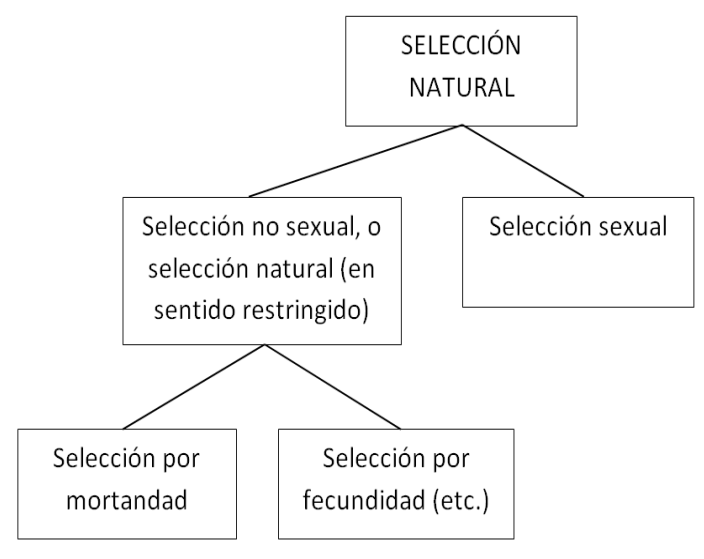

Fig. 1 - Subdivisiones de TSN según Endler (Endler 1986, p. 9).

En otras oportunidades (Ginnobili 2009, 2010a) presenté a partir de la obra de Darwin, las diferentes formas en la ley fundamental de TSN puede especializarse en diferentes leyes especiales, señalando especializaciones no tomadas en cuenta por Endler (ver fig. 2). 


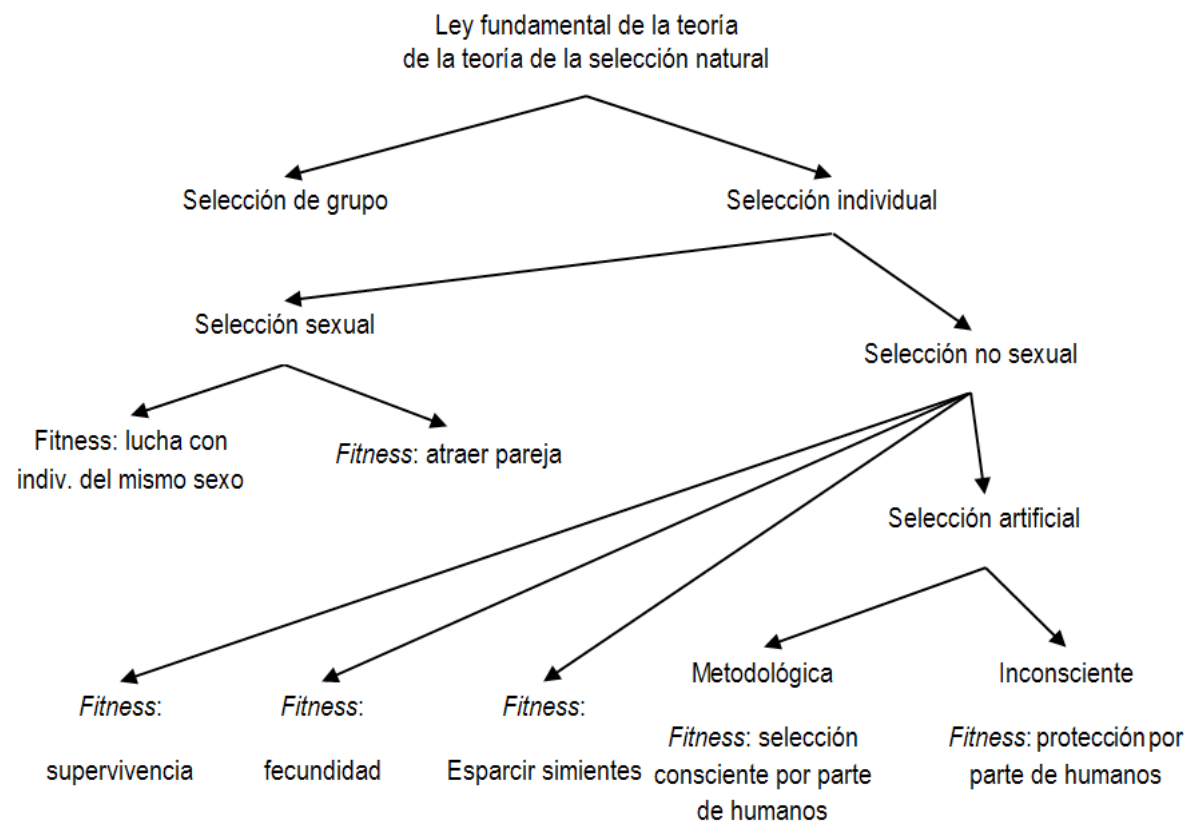

Fig. 2 - Ilustración de las diferentes formas en las que la ley fundamental de TSN puede especificarse.

Si se toman en cuenta las dos cuestiones señaladas, es decir, si se consideran todos los componentes de TSN y las diferentes leyes especiales a través de las cuales esta ley fundamental se aplica, es posible dar una respuesta más satisfactoria a la relación entre el fitness ecológico y las propiedades físicas de los organismos.

\section{RED TEÓRICA Y LA RELACIÓN DE ESPECIALIZACIÓN}

En esta sección intentaré insertar las reflexiones realizadas en el apartado anterior dentro del marco teórico estructuralista. Si bien el estructuralismo metateórico puede ser concebido esencialmente como una herramienta para reconstruir teorías científicas, ofrece una serie de conceptos metateóricos que permiten hablar de las teorías científicas de manera más precisa y sofisticada. Algunas de las características que dentro del estructuralismo metateórico se señalan como típicas de las teorías científicas, nos servirán para plantear la cuestión que estamos tratando en mejores términos.

La acepción con la que usualmente se utiliza el término «teoría», la que por ejemplo se utiliza al hablar de «la teoría de la mecánica clásica», es la 
de «red teórica». Los estructuralistas llaman «red teórica» a un conjunto de elementos teóricos con varios niveles de estratificación (Balzer, Moulines \& Sneed 1987, pp. 167-203). La estructura es ramificada y la relación entre los elementos teóricos no es deductiva, sino de especialización. Esta relación es antisimétrica, transitiva y reflexiva. Cada uno de los elementos especifica componentes no especificados en los elementos de los cuales es una especialización. Normalmente todos los elementos teóricos son especializaciones de un mismo elemento teórico, llamado «elemento teórico básico». El elemento teórico básico sería equivalente a lo que en términos presistemáticos llamamos, en los apartados anteriores, «ley fundamental». El gráfico de la figura 2 representa la red teórica de TSN, que muestra la estructura ramificada que suelen tener las redes teóricas. Los elementos teóricos especializados que pertenecen a una misma red teórica tienen el mismo marco conceptual, aunque tales conceptos van especificándose mientras se desciende en la red teórica. La red teórica de la mecánica clásica surge a partir de las diferentes especificaciones que sufre el concepto de fuerza. Las diferentes especializaciones hacen referencia a distintos tipos de fuerzas cada una con características particulares. La red teórica de TSN surge a partir de la especificación del concepto de fitness. ¿Qué tienen en común las diferentes fuerzas, por ejemplo, la fuerza de rozamiento y la fuerza de gravedad? Pues, que cumplen un papel análogo en las diferentes leyes especiales, y que son especificaciones de un mismo concepto, el concepto de fuerza (por esos son fuerzas). ¿Qué tienen en común en el caso de TSN la fecundidad y la supervivencia? Se puede dar la misma respuesta. Cumplen un papel análogo en las leyes especiales en las que aparecen (conectan la efectividad con la que un rasgo cumple una función con el éxito reproductivo del organismo que lo porta) y son especificaciones de un mismo concepto: el de fitness.

Como veíamos, la sospecha de Rosenberg, Sober y Brandon es que el fitness de los organismos se encuentra interconectado con un número heterogéneo y vasto de capacidades físicas diferentes y condiciones ambientales, volviéndose imposible establecer incluso una pequeña proporción de las conexiones nomológicas entre un fitness dado y todas las diferentes propiedades y relaciones de los organismos que lo pueden afectar. Si la presentación de TSN se completa en el modo señalado en el apartado anterior y se inserta la discusión en el marco del estructuralismo metateórico, parece posible brindar una respuesta adecuada a esta problemática al insertar las porpiedades físicas particulares de los organismos en el entramado de la red teórica de TSN. Pues, por vasto y heterogéneo que el conjunto de propiedades relevantes sea, es posible establecer relaciones nomológicas entre estos y el éxito reproductivo a través de la clasificación que la red teórica de TSN produce mediante las diferentes especificaciones de fitness. 
Queremos reconstruir o elucidar adecuadamente el tipo de explicación dada por Darwin o por Kettlewell, en donde no basta con encontrar una correlación entre ciertos tipos de organismos y su capacidad de dejar descendencia, sino que se apela a ciertas características de los organismos distintas al éxito reproductivo. Bajo la perspectiva brindada se da cuenta mejor de estas intuiciones al respecto del concepto de fitness, apelando a las herramientas del estructuralismo metateórico. La cuestión es que no es necesario apelar a una capacidad particular de los organismos para que el fitness sea distinto al éxito reproductivo diferencial. Teniendo en cuenta la versión más completa de la ley fundamental, es preciso señalar que las capacidades de los organismos pueden aparecer en dos de sus partes. La capacidad de mimetizarse, o de volar, no serían estrictamente especificaciones del fitness, sino de las diferentes funciones que pueden cumplir o satisfacer los diferentes rasgos. La razón por la que no es posible dar cuenta de este punto desde la reconstrucción ofrecida, por ejemplo, por Brandon, es que falta explicitar conceptos esenciales de TSN. Si se sostiene que la razón por la cual no se puede identificar el fitness con ninguna capacidad particular de los organismos es que el incremento en el fitness se puede deber a una mejora en la fecundidad o a una mejora en la supervivencia, como sostiene Brandon, entonces, efectivamente, la respuesta consiste en señalar que, efectivamente, el fitness no se identifica con ninguna de estas capacidades, pero sí se especifica en estas capacidades diferentes en las distintas leyes especiales de TSN. Esta respuesta no estaba disponible ni para Rosenberg ni para Brandon. Pues no toman en cuenta las diferentes especializaciones de TSN (a diferencia de Endler) y, por otra parte, carecen de una metateoría que dé cuenta de la forma en que la ley fundamental de una teoría se especializa en leyes especiales que surgen de la especificación de sus conceptos fundamentales.

\section{ConClusión}

He intentado señalar un camino que considero más adecuado para pensar el concepto de fitness ecológico, propio de TSN. Cómo he intentado mostrar, existen reconstrucciones de TSN que, por una parte, no logran explicitar todos los conceptos presupuestos en sus aplicaciones, y por otro, no dan cuenta de las diferentes formas en las que TSN se aplica a través de sus diferentes leyes especiales. He intentado mostrar en qué sentido, considerando estas dos cuestiones es posible dar cuenta del papel de las habilidades de los organismos vivos y sus propiedades físicas en una explicación seleccionista darwiniana. Al insertar esta problemática en el marco teórico estructuralista, además de presentar la cuestión de modo más claro, se muestra que TSN es una teoría con las mismas características que otras teorías de la biología o de otras disciplinas. Así como no es necesario apelar a conceptos como la superveniencia para garantizar la capacidad explicativa de la mecánica clásica, tampoco es necesario 
hacerlo para la aplicación de TSN. Basta con considerar el hecho generalizado de que las teorías unificadoras se aplican a través de sus leyes especiales. Las leyes especiales permiten ordenar el grupo enorme de habilidades en las que el fitness se instancia. Existe una cuestión no tratada en este artículo, relevante para la comprensión de cómo el fitness se calcula. Según Rosenberg, por ejemplo, el hecho de que el fitness no pueda reducirse a una habilidad particular de los organismos, implica que sólo pueda ser determinado a través de las tasas reproductivas reales de los organismos. Si se toma en cuenta (como se lo hace en la presentación de Endler como en la mía) que el fitness se especifica en conceptos como fecundidad o fertilidad, entonces se abre la puerta a que pueda ser calculado con independencia de las tasas reproductivas. En otra oportunidad he defendido que este es el caso (Ginnobili 2011a). Eso tiene consecuencias interesantes sobre el estatus de teoricidad fitness y sobre el estatus de TSN. Esto queda por fuera de las objetivos del artículo, en donde se ha intentado mostrar la importancia de presentar una versión de TSN más completa en la que se consideren conceptos que habitualmente no se explicitan y las leyes especiales en las que la ley fundamental de TSN se especializa.

\section{REFERENCIAS BIBLIOGRÁFICAS}

ARIEW, A. y R. C. LEWONTIN (2004), «The Confusions of Fitness», British Journal for the Philosophy of Science 55: 347-363

BALZER, W. y C. U.MOULINES (1996), Structuralist theory of science : focal issues, new results. Berlin: de Gruyter.

BALZER, W., C. U. MOULINES y J. D. SNEED (1987), An architectonic for science: the structuralist program. Dordrecht, Lancaster: Reidel.

BARBADILLA, A. (1990), «La estructura de la teoría de la selección natural», en A. Ruiz y M. Santos (eds.), Temas Actuales de Biología Evolutiva, Barcelona: $\mathrm{UAB}$.

BOUCHARD, F. (2011), «Darwinism without populations: a more inclusive understanding of the «Survival of the Fittest»», Studies in History and Philosophy of Biological and Biomedical Sciences 42: 106-114

BRANDON, R. (1978), «Adaptation and evolutionary theory», Studies in History and Philosophy of Science 9:181-206 ,(1980), «A Structural Description of Evolutionary Theory», PSA: Proceedings of the Biennial Meeting of the Philosophy of Science Association 1980: 427-439 , (1990), Adaptation and Enviroment. Princeton, New Yersey: Princeton University Press. 
CASANUEVA, M. (2011), «A Structuralist Reconstruction of the Mechanism of Natural Selection in Set Theory and Graph Formats», en J. Martinez Contreras y A. Ponce de León (eds.), Darwin's Evolving Legacy, México: Siglo XXI, pp. 177-192.

DAVIDSON, D. (1970), «Mental Events», en L. Foster y J. W. Swanson (eds.), Experience and theory, Amherst: University of Massachusetts Press.

DIEZ, J. A. y P. LORENZANO, eds. (2002), Desarrollos actuales de la metateoría estructuralista: Problemas y discusiones. Bernal: Universitata Rovira i Virgili Coordinación General de Investigación y Posgrado Universidad Nacional de Quilmes

ENDLER, J. A. (1986), Natural Selection in the Wild. Princeton, New Jersey: Princeton University Press.

, (1992), «Natural selection: current usages», en E. Fox Keller y E. A. Lloyd (eds.), Keywords in Evolutionary Biology, Cambridge, Mass.: Harvard University Press, pp. 220-224.

FUTUYMA, D. J. (1986), Evolutionary Biology. Second Edition. Sunderland, Massachusetts: Sinauer Associates, Inc.

GAYON, J. (1998), Darwinism's struggle for survival: heredity and the hypothesis of natural selection, Cambridge studies in philosophy and biology. Cambridge: Cambridge University Press.

GINNOBILI, S. (2009), «El poder unificador de la teoría de la selección natural», en M. C. Barboza, J. D. Avila, C. Píccoli y J. Cornaglia Fernández (eds.), 150 años después... La vigencia de la teoría evolucionista de Charles Darwin, Rosario: Universidad Nacional de Rosario, pp. 141-154.

, (2010a), «La teoría de la selección natural darwiniana», Theoria 25 (1): 37 58

, (2010b), «La teoría de la selección natural darwiniana y la genética de poblaciones», Endoxa (24): 169-184

,(2011a), «El estatus fenomenológico de la teoría de la selección natural», Ideas $y$ Valores (145): 69-86

, (2011b), Tesis doctoral: La estructura de la teoría de la selección natural Elucidación de sus conceptos fundamentales, reconstrucción de su estructura y consecuencias del análisis sobre algunas discusiones metateóricas a su alrededor. Facultad de Filosofía y Letras, Buenos Aires: Universidad de Buenos Aires.

, (2012), «Reconstrucción estructuralista de la teoría de la selección natural», Ágora. Papeles de filosofía 31 (2): 143-169

GLYMOUR, B. (2006), «Wayward Modeling: Population Genetics and Natural Selection», Philosophy of Science 73 (4): 369-389

KETTLEWELL, H. B. D. (1955), «Selection experiments on industrial melanism in the Lepidoptera», Heredity 9: 323-342

, (1956), «Further selection experiments on industrial melanism in the Lepidoptera», Heredity 10 (3): 287-301

KUHN, T. S. (1970), «Postscript-1969», en, The Structure of Scientific Revolutions, Chicago: University of Chicago Press, pp. 174-210. 
LLOYD, E. A. (1994), The Structure and Confirmation of Evolutionary Theory. New Jersey: Princeton University Press.

MATTHEN, M.y A. ARIEW (2002), «Two Ways of Thinking about Fitness and Natural Selection», Journal of Philosophy 99 (2): 55-83

MILLS, S. K. y J. H. BEATTY (1979), «The Propensity Interpretation of Fitness», Philosophy of Science 46 (2): 263-286

MOULINES, C. U. (1982), Exploraciones metacientíficas. Madrid: Alianza Editorial.

MOULINES, C. U. (1991), Pluralidad y Recursión. Madrid: Alianza Universidad.

PEACOCK, K. A. (2011), «The three faces of ecological fitness», Studies in History and Philosophy of Biological and Biomedical Sciences 42: 99-105

PEREZ, D. (1996), «Variedades de superveniencia», Manuscrito 19 (2): 165-199

PIGLIUCCI, M. y J. M. KAPLAN (2006), Making Sense of Evolution. Chicago, London: The University of Chicago Press.

ROSENBERG, A. (1978), «The Supervenience of Biological Concepts», Philosophy of Science 45 (3): 368-386 , (1983), «Fitness», The Journal of Philosophy 80 (8): 457-473

,(1985), The Structure of Biological Science. Cambridge: Cambridge University Press.

, (1994), Instrumental Biology or The Disunity of Science. Chicago and London: The university of Chicago Press.

ROSENBERG, A. y F. BOUCHARD (2009), «Fitness», en E. N. Zalta (ed.), The Stanford Encyclopedia of Philosophy.

ROSENBERG, A. y D. W. Mcshea (2008), Philosophy of Biology - A contemporany introduction. New York \& London: Routledge.

RUSE, M. (1973), The Philosophy of Biology. London: Hutchinson \& Co.

SOBER, E. (1993), The Nature of Selection. Chicago: The University of Chicago Press.

THOMPSON, P. (1989), The Structure of Biological Theories. New York: State University of New York Press.

WILLIAMS, M. B. (1970), «Deducing the consequences of evolution: A mathematical model», Journal of Theoretical Biology (29): 343-385.

SANTIAGo Ginobili es profesor la Universidad de Buenos Aires y en la Universidad Nacional de Quilmes (Argentina).

Lineas de investigación:

Filosofía de la ciencia, filosofía de la biología

Publicaciones recientes:

(2011) «Función como concepto teórico», en Scientiae Studia, vol. 9, nº 4, São Paulo, 
Brasil, pp. 847-880, ISSN 1678-3166. Accesible en http://www.scielo.br/scielo.php?pid=S1678-31662011000400006\&script=sci_arttext. (2012) «Reconstrucción estructuralista de la teoría de la selección natural», en Agora 31(2), pp.143-169, ISSN 0211-6642.

Dirección electrónica: santi75@gmail.com 